\title{
Sequential Contribution of Parenchymal and Neural Stem Cell-Derived Oligodendrocyte Precursor Cells toward Remyelination
}

\author{
David R. Serwanski ${ }^{1}$, Andrew L. Rasmussen ${ }^{1}$, Christopher B. Brunquell ${ }^{1}$, Scott S. Perkins ${ }^{1}$ \\ and Akiko Nishiyama ${ }^{1,2,3, *}$ \\ 1 Department of Physiology and Neurobiology, University of Connecticut, 75 North Eagleville Road, Storrs, \\ CT 06269-3156, USA; drsbeek@gmail.com (D.R.S.); andy.l.rasmussen@gmail.com (A.L.R.); \\ christopherbrunquell@gmail.com (C.B.B.); scoperk11@gmail.com (S.S.P.) \\ 2 Institute for Systems Genomics, University of Connecticut, Storrs, CT 06269, USA \\ 3 Institute for Brain and Cognitive Science, University of Connecticut, Storrs, CT 06269, USA \\ * Correspondence: akiko.nishiyama@uconn.edu
}

Received: 1 May 2018; Accepted: 4 June 2018; Published: 12 June 2018

\begin{abstract}
In the adult mammalian forebrain, oligodendrocyte precursor cells (OPCs), also known as NG2 glia are distributed ubiquitously throughout the gray and white matter. They remain proliferative and continuously generate myelinating oligodendrocytes throughout life. In response to a demyelinating insult, OPCs proliferate rapidly and differentiate into oligodendrocytes which contribute to myelin repair. In addition to OPCs, neural stem cells (NSCs) in the subventricular zone (SVZ) also contribute to remyelinating oligodendrocytes, particularly in demyelinated lesions in the vicinity of the SVZ, such as the corpus callosum. To determine the relative contribution of local OPCs and NSC-derived cells toward myelin repair, we performed genetic fate mapping of OPCs and NSCs and compared their ability to generate oligodendrocytes after acute demyelination in the corpus callosum created by local injection of $\alpha$-lysophosphatidylcholine (LPC). We have found that local OPCs responded rapidly to acute demyelination, expanded in the lesion within seven days, and produced oligodendrocytes by two weeks after lesioning. By contrast, NSC-derived NG2 cells did not significantly increase in the lesion until four weeks after demyelination and generated fewer oligodendrocytes than parenchymal OPCs. These observations suggest that local OPCs could function as the primary responders to repair acutely demyelinated lesion, and that NSCs in the SVZ contribute to repopulating OPCs following their depletion due to oligodendrocyte differentiation.
\end{abstract}

Keywords: demyelination; oligodendrocyte precursor; myelin; subventricular zone; NG2; neural stem cell

\section{Introduction}

In the mammalian central nervous system (CNS), oligodendrocyte precursor cells (OPCs), also known as NG2 glia or polydendrocytes, represent a fourth major glial cell population that persists in the adult and continue to proliferate and generate myelinating oligodendrocytes throughout life, even after developmental myelination is largely completed (reviewed in [1,2]). Studies in the adult rat spinal cord have shown that OPCs proliferate in response to acute demyelination, and that the proliferated cells differentiate into oligodendrocytes [3-5]. Oligodendrocyte precursor cells in the telencephalon also contribute to remyelination of acute and chronic demyelinated lesions $[3,6,7]$. Genetic fate mapping has allowed more precise determination of the fate of OPCs under normal conditions [8-11] and established that the postnatal fate of OPCs is restricted to oligodendrocytes. 
This approach was also used to confirm that OPCs are an important source of remyelinating oligodendrocytes that repair demyelinated lesions [12,13].

In addition to local proliferating NG2 glia, the adult brain also contains neural stem cells (NSCs) that reside in the subventricular zone (SVZ). Neural stem cells in the adult SVZ pass through a transit-amplifying progenitor stage as they differentiate into neuroblasts that migrate through the rostral migratory stream (RMS) into the olfactory bulb, and their multipotential ability to generate astrocytes as well as neurons has been well characterized [14,15]. When demyelination occurs in the vicinity of the SVZ, cells in the SVZ become mobilized and may contribute to remyelination [16-18]. The availability of genetic fate mapping tools to identify the fate of OPCs and NSCs have allowed a direct comparison of the contribution of local OPCs and SVZ-derived cells toward myelin repair, and several studies were published recently with varying results. While two studies suggest a robust ability of NSCs to generate remyelinating oligodendrocytes $[19,20]$, another recent study suggests that although SVZ cells proliferate in response to acute demyelination, they are unable to produce oligodendrocytes that contribute to myelin repair [21]. We have used $\mathrm{Tg}\left(\mathrm{Cspg} 4\right.$-creER $\left.{ }^{\mathrm{TM}}\right)$ and $\mathrm{Tg}\left(\mathrm{Nes}-\mathrm{creER}^{\mathrm{T} 2}\right)$ transgenic mice crossed to a reporter line and have compared the ability of local OPCs and nestin+ NSCs to generate oligodendrocytes in response to acute demyelination caused by $\alpha$-lysophosphatidylcholine (LPC) injection into the adult corpus callosum. We show that local OPCs generate oligodendrocytes more rapidly than NSCs, whereas NSCs contribute to repopulating OPCs in the corpus callosum after myelin repair.

\section{Materials and Methods}

\subsection{Animals}

A bacterial artificial chromosome (BAC) transgenic mouse line expressing tamoxifen-inducible Cre in OPCs (Tg(Cspg4-creER $\left.{ }^{\mathrm{TM}}\right)$ [9]; Jackson Laboratory stock \#008538, Bar Harbor, ME, USA) was crossed to the cre reporter gt(ROSA)26Sor ${ }^{\text {tm1(EYFP) }}$ (YFP) [22] (Jackson Laboratory stock \#006148) and maintained as double homozygotes. $\mathrm{Tg}\left(\mathrm{Nes}-\mathrm{creER}^{\mathrm{T} 2}\right)$ mice were obtained from Dr. Amelia Eisch (University of Pennsylvania, Philadelphia, PA, USA) [23] and also crossed to YFP mice. Cre was induced by four consecutive days of intraperitoneal injection of $1 \mathrm{mg}$ of 4-hydroxytamoxifen (4OHT, Sigma H-7904, St. Louis, MO, USA) every $12 \mathrm{~h}$, as previously described [9].

To create acute demyelinating lesions, $1 \mu \mathrm{L}$ of $2 \%$ LPC in phosphate-buffered saline (PBS) was stereotaxically injected into the right rostral corpus callosum using a Hamilton syringe at the coordinates ( $-1.3 \mathrm{~mm}$ from the bregma, $1 \mathrm{~mm}$ lateral, and $1.7 \mathrm{~mm}$ from the surface of the skull). Mice were sacrificed at 7,14 , and 28 days after LPC injection $(7,14$, and $28 \mathrm{dpl})$ by intracardiac perfusion of $4 \%$ paraformaldehyde in $0.1 \mathrm{M}$ sodium phosphate buffer, $\mathrm{pH} 7.4$, containing $0.1 \mathrm{M}$ L-lysine and $0.01 \mathrm{M}$ sodium metaperiodate. Brains were isolated and post- fixed in the same fixative for $2 \mathrm{~h}$ at 4 ${ }^{\circ} \mathrm{C}$. For NG2creER;YFP mice, LPC injection was performed three days after the last $4 \mathrm{OHT}$ injection. For $\mathrm{Tg}\left(\mathrm{Nes}_{-} \mathrm{creER}^{\mathrm{T} 2}\right)$ :gt(ROSA)26Sor ${ }^{\text {tm1(EYFP) }}{ }^{\text {mice, }}$ LPC injection was performed 28 days after $4 \mathrm{OHT}$ injection to allow for greater accumulation of YFP+ cells in the SVZ.

All animal procedures were approved by the Institutional Animal Care and Usage Committee in a protocol A16-018 "NG2 cells in the neural network" from June 24, 2016 through June 23, 2019.

\subsection{Tissue Processing and Immunohistochemistry}

Serial $50 \mu \mathrm{m}$ coronal sections were cut on a vibratome (Leica VTS1000, Leica Biosystems Inc., Buffalo Grove, IL, USA) and stored at $-20^{\circ} \mathrm{C}$ in 98 -well plates in cryostorage solution consisting of 10 g of polyvinylpyrolidone, $500 \mathrm{~mL} 0.2 \mathrm{M}$ sodium phosphate buffer, $\mathrm{pH} 7.4,300 \mathrm{~g}$ sucrose, and $300 \mathrm{~mL}$ ethylene glycol in $1 \mathrm{~L}$. Sections were processed for immunohistochemistry as previously described [9]. The primary antibodies used are listed in Table 1. Secondary antibodies were Alexa 488-labeled anti-chick antibody (1:1000), Cy3-labeled anti-mouse, rabbit, or goat (1:200), and Cy5- (1:100) or Alexa 647-labeled (1:200) anti-mouse, rabbit, or goat antibodies from Jackson ImmunoResearch (West 
Grove, PA, USA). Five or six sections from each mouse that were $600 \mu \mathrm{m}$ apart were labeled with the antibodies and mounted with Vectashield containing 4',6-diamidino-2-phenylindole dihydrochloride (DAPI, Vector Labs, Burlingame, CA, USA). Stained sections were analyzed on Leica TCS SP2 or SP8 confocal microscope and Zeiss Axiovert $200 \mathrm{M}$ with ORCA ER camera and apotome (Carl Zeiss Microscopy, Jena, Germany).

Table 1. Primary antibodies used.

\begin{tabular}{cccc}
\hline Antibody & Host Species & Source & Dilution \\
\hline Dcx & Rabbit & Cell Signaling Technology (Danvers, MA, USA) & $1: 300$ \\
NG2 & Rabbit & EMD Millipore (Burlington, MA, USA) & $1: 500$ \\
Pdgfra & Goat & R\&D Systems (Minneapolis, MN, USA) & $1: 1000$ \\
CC1 (Quaking 7) & Mouse & EMD Millipore (Burlington, MA, USA) & $1: 100$ \\
MBP, smi99 antibody & Mouse & Covance (Princeton, NJ, USA) & $1: 3000$ \\
Smi32 & Mouse & Covance (Princeton, NJ, USA) & $1: 1000$ \\
GFP & Chick & Aves Labs (Tigard, OR, USA) & $1: 1000$ \\
Olig2 & Mouse & EMD Millipore (Burlington, MA, USA) & $1: 1000$ \\
Olig2 & Rabbit & Novus Biologicals (Littleton, CO, USA) & $1: 1000$ \\
PSA-NCAM, 12E3 antibody & Mouse & Dr. Tatsunori Seki (Tokyo Medical University, Tokyo, Japan) & $1: 1000$ \\
GFAP & Rabbit & DAKO-Agilent (Santa Clara, CA, USA) & $1: 2000$ \\
\hline
\end{tabular}

Dcx: Doublecortin; Pdgfra: Platelet-derived growth factor receptor $\alpha$; MBP: Myelin basic protein; Smi32: Non-phosphorylated neurofilaments; GFP: Green fluorescent protein; Olig2: Oligodendrocyte transcription factor 2; PSA-NCAM: Polysialic acid-neural cell adhesion molecule; GFAP: Glial fibrillary acidic protein.

\subsection{Cell Quantification}

Demyelinated lesions were assessed by a combination of immunolabeling for myelin basic protein (MBP) and non-phosphorylated neurofilaments. For quantification, a series of tiled confocal $z$-stack images were collected over a $z$-distance of 20-30 $\mu \mathrm{m}$, encompassing the lesion from at least two sections for each animal. Cell numbers were estimated by scoring each YFP+ cell for the expression of specific antigens within a defined area for each section. The area for quantification was defined as the area that lacked MBP immunofluorescence in adjacent sections for the early time points. For later time points, the lesioned and repaired areas were defined as areas that contained dense reactive NG2 glia and astrocytes. To obtain the density of the cells, the area from which cell numbers were obtained was calculated using ImageJ [24] and multiplied by the thickness of the $z$-stack.

\subsection{Statistical Analysis}

Quantification results are expressed as mean \pm standard deviation. Statistical analyses were performed using two-way analysis of variance (ANOVA) with uncorrected Fisher's least significant difference (LSD) test for the quantification of \% YFP+ cells that were NG2+ or CC1+ and the density of YFP+ NG2+ and YFP+ CC1+ cells. Student's t-test (two-way, unpaired) was used for the quantification of the percentage of $\mathrm{CC} 1+$ cells derived from NG2+ or nestin+ precursor cells over 14 days. Sample sizes ranged from three to four.

\section{Results}

\subsection{Evolution of LPC-Induced Demyelinated Lesion}

In the normal adult mouse corpus callosum, MBP was robustly detected in the corpus callosum, and there was little detectable non-phosphorylated neurofilaments, with the exception of axons in the cingulate cortex (Figure 1A-C). Injection of LPC into the corpus callosum resulted in focal demyelination, characterized by a well demarcated loss of MBP immunoreactivity at 7 days after lesioning (dpl) (Figure 1D-F, arrowheads in D), accompanied by increased immunoreactivity for non-phosphorylated neurofilaments, which have been shown to increase in demyelinated axons [25]. By $14 \mathrm{dpl}$, the area of demyelinated lesion had decreased, and a substantial amount of myelin had been regenerated, while non-phosphorylated neurofilaments were still present. By $28 \mathrm{dpl}$, the lesion was indistinguishable from the surrounding myelinated region in the majority of the animals. The evolution of the lesion was consistent with previously published reports (for example, [26]). 


\subsection{Contribution of Local OPCs to Remyelinating Oligodendrocytes}

To investigate the extent to which local OPCs contribute to remyelination, we used $\mathrm{Tg}$ (Cspg4-creER ${ }^{\mathrm{TM}}$;gt(ROSA)26Sor ${ }^{\text {tm1(EYFP) }}$ (NG2-YFP) double transgenic mice. The fate of local OPCs was followed during the course of demyelination and remyelination by activating cre-mediated recombination and YFP expression in OPCs 3-4 days prior to LPC injection (Figure 2A). One day after the last tamoxifen injection, $40-50 \%$ of OPCs in the corpus callosum were YFP+ [9]. We induced cre before LPC injection to avoid activating YFP expression in macrophages that could also express NG2 [27]. This regimen also minimized labeling of SVZ progenitor cells that were mobilized and upregulated NG2 expression after demyelination.

To determine the dynamics of oligodendrocyte differentiation from parenchymal OPCs, we determined the percentage of YFP+ cells that were NG2+ oligodendrocyte precursor cells or $\mathrm{CC} 1+$ differentiated oligodendrocytes at 7,14 , and $28 \mathrm{dpl}$. At $7 \mathrm{dpl}$, when there was a well-defined demyelinated lesion characterized by a lack of MBP reactivity, clusters of YFP+ cells were seen in and around the lesion suggestive of local proliferation (Figure 2B,C). Among the $\mathrm{YFP}+$ cells, $80 \%$ were NG2+, while only $10 \%$ were CC1+ oligodendrocytes (Figure $2 \mathrm{E}, \mathrm{K}, \mathrm{L}$ ). By $14 \mathrm{dpl}$, the proportion of YFP+ cells that were NG2+ decreased to $62 \%$, while the proportion of YFP+ cells that were CC1+ increased to $44 \%$ (Figure 2D,E,K,L), and clusters of YFP+ CC1+ cells were found inside the lesion (Figure 2F,G). Some YFP+ cells expressed both NG2 and CC1, which suggests that OPCs were rapidly differentiating into $\mathrm{CC} 1+$ oligodendrocytes. Outside the lesion, both YFP+ NG2+ cells and YFP+ CC1+ oligodendrocytes were detected as well (Figure $2 \mathrm{H}$ ). The proportion of YFP+ cells that were $\mathrm{CC} 1+$ in and around the lesion at $14 \mathrm{dpl}$ was more than 4 -fold greater than that at $7 \mathrm{dpl}$, suggesting that OPCs that were recruited to the lesion were undergoing oligodendrocyte differentiation. At $28 \mathrm{dpl}$, the proportion of YFP+ cells that were NG2+ remained at 63\%, while the proportion of YFP+ cells that were $\mathrm{CC} 1+$ decreased slightly to $30 \%$ (Figure 2I-L).

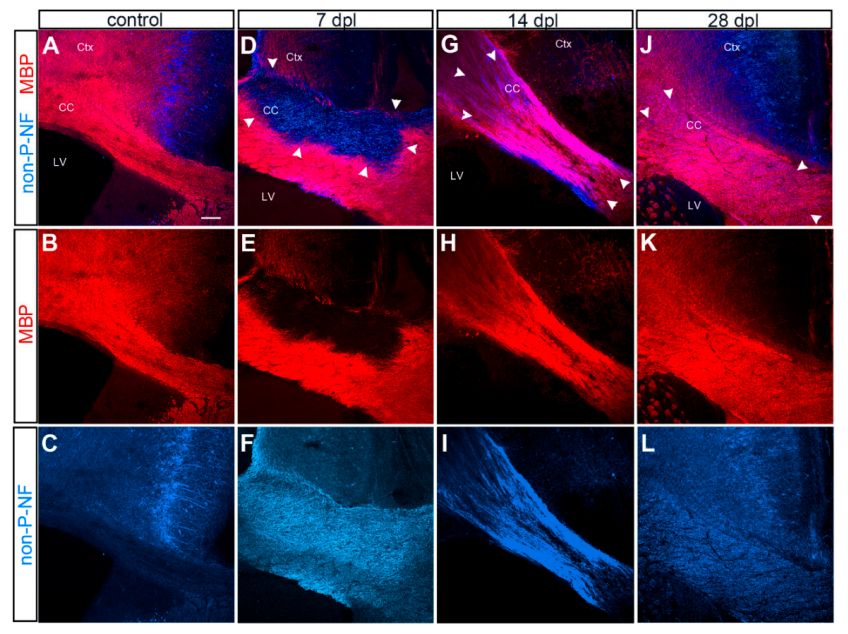

Figure 1. Evolution of $\alpha$-lysophosphatidylcholine (LPC)-induced demyelinated lesion. Immunofluorescence labeling for myelin basic protein (MBP) and non-phosphorylated neurofilaments. (A-C) Control unlesioned brain. Intact MBP+ myelin in the corpus callosum. Non-phosphorylated neurofilaments are restricted to the neurons in the cingulate cortex. Ctx: cortex, CC: corpus callosum, LV: lateral ventricle. (D-F) Demyelinated corpus callosum at 7 days post lesioning (dpl) showing a well-defined lesion lacking MBP and upregulated non-phosphorylated neurofilaments. Boundary of the lesion is indicated by arrowheads. (G-I) Demyelinated corpus callosum at $14 \mathrm{dpl}$ showing partial remyelination, characterized by uneven MBP labeling and persistent presence non-phosphorylated neurofilaments. (J-L) Remyelinated corpus callosum at $28 \mathrm{dpl}$ showing uniform MBP labeling and reduced levels of non-phosphorylated neurofilaments, though they are higher than unlesioned corpus callosum. Scale bar: $100 \mu \mathrm{m}$. 
When we examined the density of total YFP+ cells and YFP+ NG2+ cells, there was no significant difference between 7 and $14 \mathrm{dpl}$, but a 3-fold increase at $28 \mathrm{dpl}$ (Figures 2J and 3P,Q). By contrast, the density of YFP+CC1+ cells continued to rise from 7 to $28 \mathrm{dpl}$ (Figure 3R). This suggests that after demyelination local OPCs already proliferated by $7 \mathrm{dpl}$ and actively generated oligodendrocytes over the course of four weeks. The increase in the density of YFP+ NG2+ cells at $28 \mathrm{dpl}$ likely reflects continued proliferation of local OPCs that existed prior to demyelination, as well as migration of OPCs from the surrounding areas into the lesion and the surrounding corpus callosum.

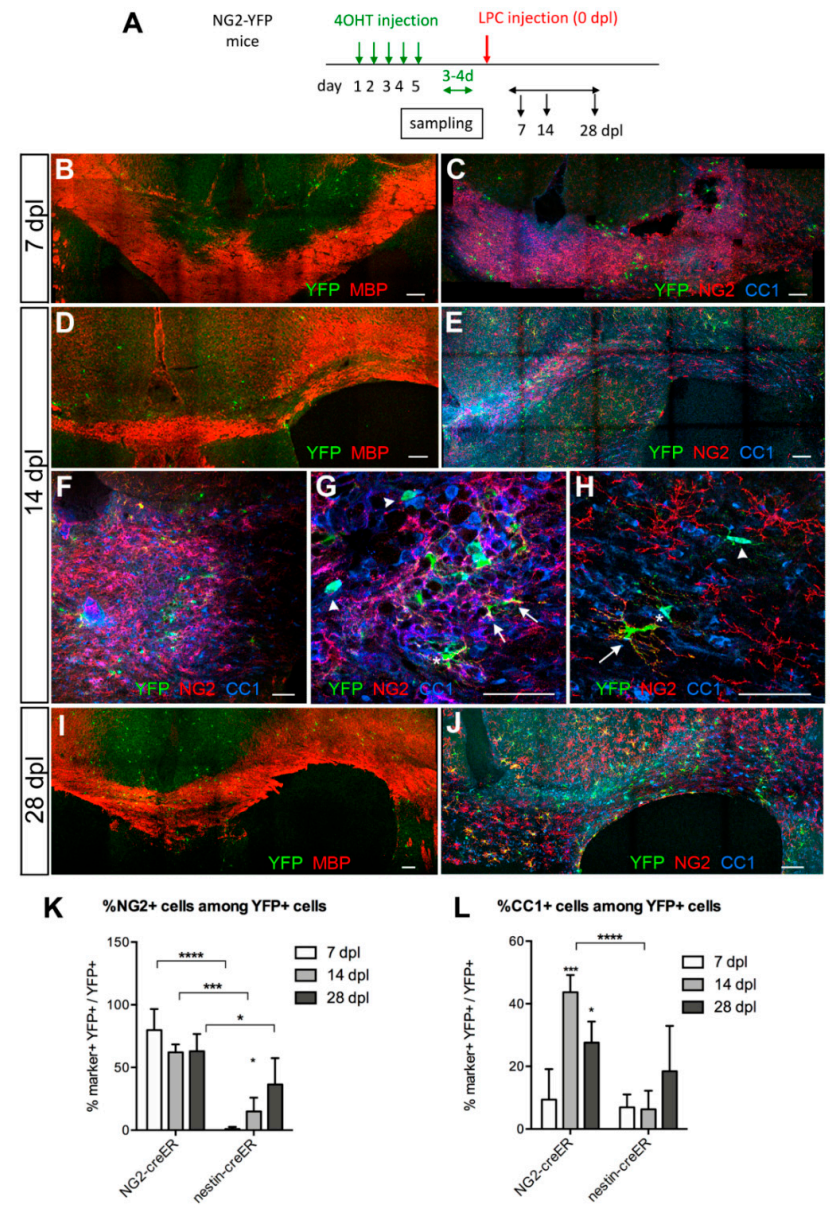

Figure 2. Response of local oligodendrocyte precursor cells (OPCs) to LPC-induced demyelination in the corpus callosum. (A) Scheme showing the experimental outline. (B,C) Lesion at $7 \mathrm{dpl}$. Low magnification images of immunolabeling for MBP and yellow fluorescent protein (YFP) showing an area of demyelination (B) and immunolabeling for YFP, NG2, and CC1 showing scattered YFP+NG2+ cells in the lesion (C). (D-H) Lesion at $14 \mathrm{dpl}$. Low magnification images of immunolabeling for MBP and YFP showing partially remyelinated lesion (D), characterized by uneven MBP staining, and immunolabeling for YFP, NG2, and CC1 showing increased number of YFP+ cells in the lesion (E,F). Higher magnification shows a significant proportion of YFP+ cells express CC1 (arrowheads), while other YFP+ cells are NG2+ (arrows). Some YFP+ cells express both NG2 and CC1 (asterisks) at varying ratios. $(\mathrm{G})$ is a higher magnification of the lesion. $(\mathbf{H})$ is from a site further away from the lesion. (I,J) Lesion at $28 \mathrm{dpl}$. Immunolabeling for MBP and YFP shows largely repaired lesion (I) and a cluster of YFP $+\mathrm{CC} 1+$ cells in the center of the repaired lesion while YFP+ NG2+ cells are seen at the periphery $(\mathbf{J})$. Scale bars: $100 \mu \mathrm{m}$ for $(\mathbf{B}-\mathbf{F})$ and $(\mathbf{I}, \mathbf{J}) ; 50 \mu \mathrm{m}$ for $(\mathbf{F}-\mathbf{H})$. $(\mathbf{K}, \mathbf{L})$ The proportion of YFP+ cells that were $\mathrm{NG} 2+(\mathbf{K})$ or $\mathrm{CC} 1+(\mathbf{L})$ in $\mathrm{Tg}\left(\mathrm{Cspg} 4-\mathrm{creER}^{\mathrm{TM}}\right.$;gt(ROSA)26Sor ${ }^{\text {tm1(EYFP) }}$ (NG2-YFP) and

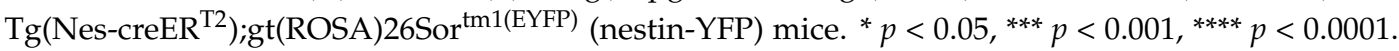
$n=3$, two-way ANOVA, uncorrected Fisher's least significant difference (LSD) test. 


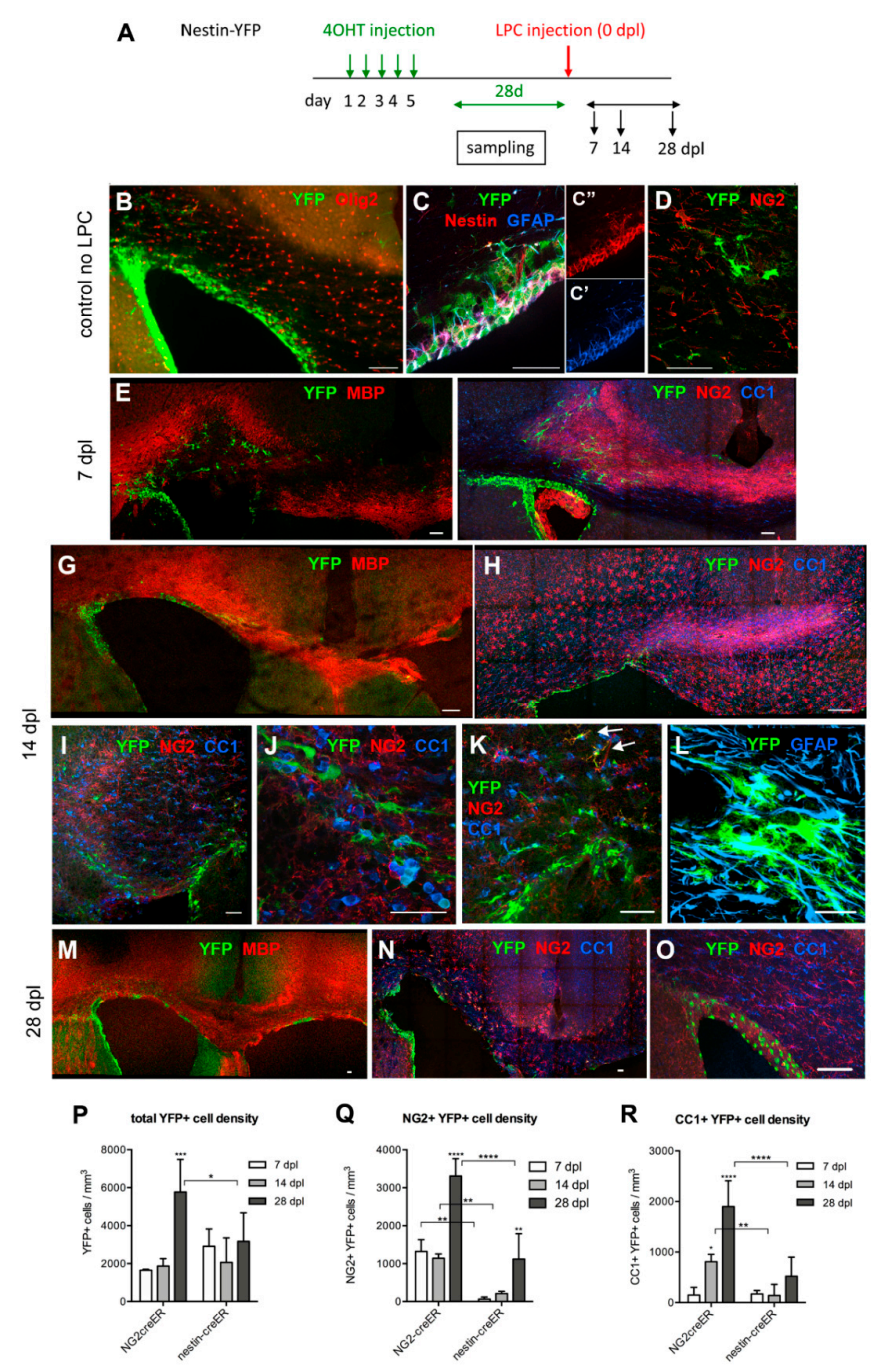

Figure 3. Response of subventricular zone (SVZ) cells to LPC-induced demyelination in the corpus callosum. (A) Scheme showing the experimental outline. (B-D) The distribution and phenotype of YFP+ cells prior to LPC injection. The majority of the YFP+ cells are found in the SVZ (B) and very few of the YFP+ cells expressed Olig2. The majority of the YFP+ cells expressed nestin and GFAP (C) but not NG2 (D). (E,F) Lesion at $7 \mathrm{dpl}$. Low magnification images of MBP and YFP immunolabeling showing an area of demyelination with YFP+ cells mostly above the lateral ventricle (E) and immunolabeling for YFP, NG2, and CC1 showing that most of the YFP+ cells are neither NG2+ nor CC1+. There is strongly upregulated NG2 immunoreactivity throughout the lesion. Note that YFP+ cells line the SVZ. Some YFP+ cells appear to be migrating toward the needle track (arrows). (G-L) Lesion at $14 \mathrm{dpl}$. Low magnification of immunolabeling for MBP and YFP showing partially remyelinated lesion (G) and immunolabeling for YFP, NG2, and CC1 showing a slightly increased number of YFP+ cells in the lesion $(\mathbf{H})$. Higher magnification shows that most of the YFP+ cells were confined to the lesion border (I). Higher magnification of I shows that most of the YFP+ cells did not express NG2 or CC1. (J) A region from the periphery of the lesion above the lateral angle of SVZ showing two YFP+ NG2+ cells (arrowheads). Some of the large, YFP+ cells were glial fibrillary acidic protein (GFAP)+ (L). (M-O) Lesion at $28 \mathrm{dpl}$. YFP and MBP labeling show remyelinated lesion with increased YFP+ cells in the lesion $(\mathbf{M})$ and that the majority of the YFP+ cells were NG2+ $(\mathbf{N})$. (O) Control mouse injected with PBS and stained for YFP, NG2, and CC1 at $28 \mathrm{dpl}$, showing that the majority of the YFP+ cells are confined to the SVZ and few YFP+ cells were detectable in the corpus callosum. Scale bar: $50 \mu \mathrm{m}$. (P-R) The density of YFP+ cells (P), YFP+ NG2+ cells (Q), and YFP+ CC1+ cells at 7, 14, and $28 \mathrm{dpl}$ in NG2-YFP and nestin-YFP mice. ${ }^{*} p<0.05,{ }^{* *} p<0.01,{ }^{* * *} p<0.001,{ }^{* * *} p<0.0001 . n=3-4$, two-way ANOVA, uncorrected Fisher's LSD. 


\subsection{Contribution of SVZ-Derived Cells to Remyelinating Oligodendrocytes}

Distribution and Phenotype of YFP+ Cells in Tg(Nes-creER ${ }^{\mathrm{T} 2}$ );gt(ROSA)26Sor ${ }^{\text {tm1(EYFP) }}{ }^{\text {Mice }}$

To assess the contribution of SVZ progenitor cells to remyelination, we performed a similar experiment in $\mathrm{Tg}\left(\mathrm{Nes}-\mathrm{creER}^{\mathrm{T} 2}\right.$ );gt(ROSA)26Sor ${ }^{\text {tm1(EYFP) }}$ (nestin-YFP) mice. We induced YFP in nestin+ progenitor cells in the SVZ 28 days prior to LPC injection, as the YFP labeling efficiency was too low immediately after tamoxifen injection (Figure 3A). At 28 days after tamoxifen injection, YFP expression was detected in $30-50 \%$ of nestin + cells in dorsal and dorsolateral SVZ, as well as in medial and latera SVZ and the rostral migratory stream (RMS). Thus, the YFP labeling efficiency among nestin+ NSCs with this induction protocol was comparable to the efficiency among OPCs in NG2cre-YFP mice. In this study, we focused on YFP+ cells in the dorsal and dorsolateral SVZ and the corpus callosum of nestin-YFP mice. Almost 90\% of the YFP+ cells resided in the SVZ, where they expressed nestin and glial fibrillary acidic protein (GFAP), two proteins known to be expressed by NSCs in the SVZ (Doetsch et al., 1999) (Figure 3B,C). A small fraction of YFP+ cells in the SVZ expressed the oligodendrocyte transcription factor Olig2, and most of the Olig2+ YFP+ cells were located in the outer layers of the SVZ. Of the YFP+ cells, $11.7 \%$ were in the corpus callosum, mostly above the dorsolateral angle of the SVZ (Figure 3B, upper left). Among the YFP+ cells in the corpus callosum, 64\% were GFAP+, $47 \%$ were nestin+, and $77 \%$ were positive for the polysialylated form of neural cell adhesion molecule (PSA-NCAM), which is known to be expressed by neuroblasts and neural progenitor cells in the neurogenic niches of the SVZ and dentate subgranular zone [28,29], as well as on growing axons that are defasciculating at the target [30,31]. Among the YFP+ cells in the corpus callosum, $7.5 \%$ expressed NG2 (Figure 3D), and only $0.8 \%$ of the total YFP+ cells in the corpus callosum and in the dorsal and dorsolateral SVZ expressed NG2 prior to LPC injection.

\subsection{Response of Nestin+ SVZ Cells to Acute Demyelination}

To examine whether nestin + SVZ progenitor cells generated OPCs that were recruited to acutely demyelinated lesions in the corpus callosum, we examined the changes in YFP+ NG2+ and YFP+ $\mathrm{CC} 1+$ cells in nestin-YFP mice over the course of four weeks after LPC injection. At the time of peak demyelination at $7 \mathrm{dpl}$, we saw an infiltration of YFP+ cells in the demyelinated corpus callosum (Figure 3E). In addition to scattered YFP+ cells in the demyelinated corpus callosum, there was a line of YFP+ cells that extended into the injection site in the more rostral sections that had the needle track (e.g., Figure 3F, arrowheads). Fewer than 3\% of the YFP+ cells were NG2+ (Figures 2K and 3F), in stark contrast to NG2-YFP mice in which $80 \%$ of the YFP+ cells were NG2+ (Figure 2K).

By $14 \mathrm{dpl}, 18 \%$ of the YFP+ cells had become NG2+, but the density of YFP+ NG2+ cells remained less than one-fourth of that in NG2-YFP mice (Figure 3G,H,Q). Many of the YFP+ cells, including those found in the lesion, had large polygonal cell bodies that were morphologically distinct from oligodendrocyte lineage cells, and some expressed GFAP (Figure 3J-L). Some YFP+ NG2+ cells with typical polydendrocyte morphology were found at the border of the lesion (Figure 3K, upper right). The percentage of YFP+ CC1+ oligodendrocytes in nestin-YFP mice did not increase from $7 \mathrm{dpl}$ to $14 \mathrm{dpl}$ (Figure 2L). Neither did the density of YFP+ CC1+ cells increase from 7 to $14 \mathrm{dpl}$ (Figure 3R) and remained 5.7-fold lower than that in NG2-YFP mice.

At $28 \mathrm{dpl}$, the most notable change was that the density of YFP+ NG2+ cells in nestin-YFP mice increased 5.3-fold over that at $14 \mathrm{dpl}$ (Figure 3Q). The average density of YFP+ CC1+ oligodendrocytes in these mice at $28 \mathrm{dpl}$ was three-fold higher than at $14 \mathrm{dpl}$ but did not reach statistical significance $(p=0.0876)$ and remained 3.6-fold lower than the density of YFP+CC1+ cells in NG2-YFP mice at $28 \mathrm{dpl}$. Thus, between 14 and $28 \mathrm{dpl}$, NSC-derived cells seemed to be most actively generating OPCs, while the parenchymal OPCs were actively producing oligodendrocytes between 7 and $14 \mathrm{dpl}$, having expanded the precursor population earlier, by $7 \mathrm{dpl}$. The density of NSC-derived OPCs in the lesion at $28 \mathrm{dpl}$ reached a comparable level to that of OPCs derived from the local population seen at 7 and $14 \mathrm{dpl}$. These observations indicate that after acute demyelination, local parenchymal OPCs initially 
responded more rapidly and robustly than SVZ-derived cells to produce oligodendrocytes, and the response of NSCs lagged behind by $2-3$ weeks.

To further evaluate the contribution of parenchymal OPCs and NSC-derived cells in the supply of oligodendrocytes to the lesion, we estimated the proportion of total CC1+ oligodendrocytes in the dorsal aspect of the corpus callosum that were generated from parenchymal OPCs or NSCs at $14 \mathrm{dpl}$, as previously estimated in the normal adult corpus callosum $[9,11]$. We obtained the percentage of $\mathrm{CC} 1+$ oligodendrocytes that were YFP+ in NG2-YFP and nestin-YFP mice at $14 \mathrm{dpl}$ and adjusted for the recombination efficiency in the respective mice. We found that $0.222 \%$ and $0.060 \%$ of CC1+ oligodendrocytes were generated from local OPCs and nestin+ NSCs, respectively, during the two weeks after demyelination. Thus, NG2-YFP mice had generated 4.3 times more CC1+ oligodendrocytes than nestin-YFP mice $(p=0.0188)$. This corroborates the other quantifications and indicates that after acute demyelination, local OPCs were more likely to contribute to remyelinating oligodendrocytes than SVZ-derived oligodendrocyte lineage cells.

The density of total YFP+ cells and YFP+ NG2+ cells in nestin-YFP mice was more than two-fold greater at $28 \mathrm{dpl}$ than at $14 \mathrm{dpl}$. In contrast to clusters of YFP+ cells found at 7 and $14 \mathrm{dpl}$, many YFP+ $\mathrm{NG} 2+$ cells at $28 \mathrm{dpl}$ were distributed more uniformly throughout the remyelinated and neighboring corpus callosum (Figure 3M,N). In PBS-injected mice, very few YFP+ cells were detected in the corpus callosum, similar to uninjured mice prior to LPC injection (Figure 3O). This suggests that demyelination triggered SVZ cells to generate oligodendrocyte lineage cells, and that the SVZ-derived cells were likely to be contributing to the replenishment of the OPC population that had been depleted due to their differentiation into remyelinating oligodendrocytes.

\subsection{Transient Migration of NSC-Derived Neuroblasts into Corpus Callosum after Demyelination}

We noticed that the dorsal SVZ was thicker in LPC-injected mice than in control PBS-injected mice. When we measured the height of the dorsal SVZ at $28 \mathrm{dpl}$, it was approximately two-fold greater in LPC-injected mice compared to PBS-injected control mice (Figure 4A,B), suggesting that there was sustained proliferative activity in the SVZ after remyelination had occurred. We examined the cellular composition in the dorsal SVZ that was expanded after demyelination. Previous studies had described PSA-NCAM+ and doublecortin (Dcx)+cells that emerged from the SVZ into acutely demyelinated corpus callosum [16,18,32]. In the SVZ at $14 \mathrm{dpl}$, we also found large clusters of PSA-NCAM+ and Dcx + cells, many of which co-expressed the two antigens and had small, round, or oval cell bodies and long slender processes (Figure 4C,D). Some of them were also YFP+ in nestin-YFP mice. These YFP+ cells had a distinct morphology from flat polygonal NSCs that were YFP+ nestin+, suggesting that they were progeny of the NSCs.

In the corpus callosum of control PBS-injected mice, there were very few PSA-NCAM+ Dcx+ cells at $14 \mathrm{dpl}$ (Figure 4E). In LPC-injected mice, a larger number of PSA-NCAM+ Dcx+ cells were seen migrating into the corpus callosum, mostly from the dorsolateral angle of the SVZ (Figure 4F-H). Their processes were oriented parallel to the axons in the corpus callosum, and many had the typical unipolar tadpole shape, as previously described $[16,18]$, consistent with their neuroblast identity (see Figure $4 \mathrm{H}$, top left cell that is also YFP+). 

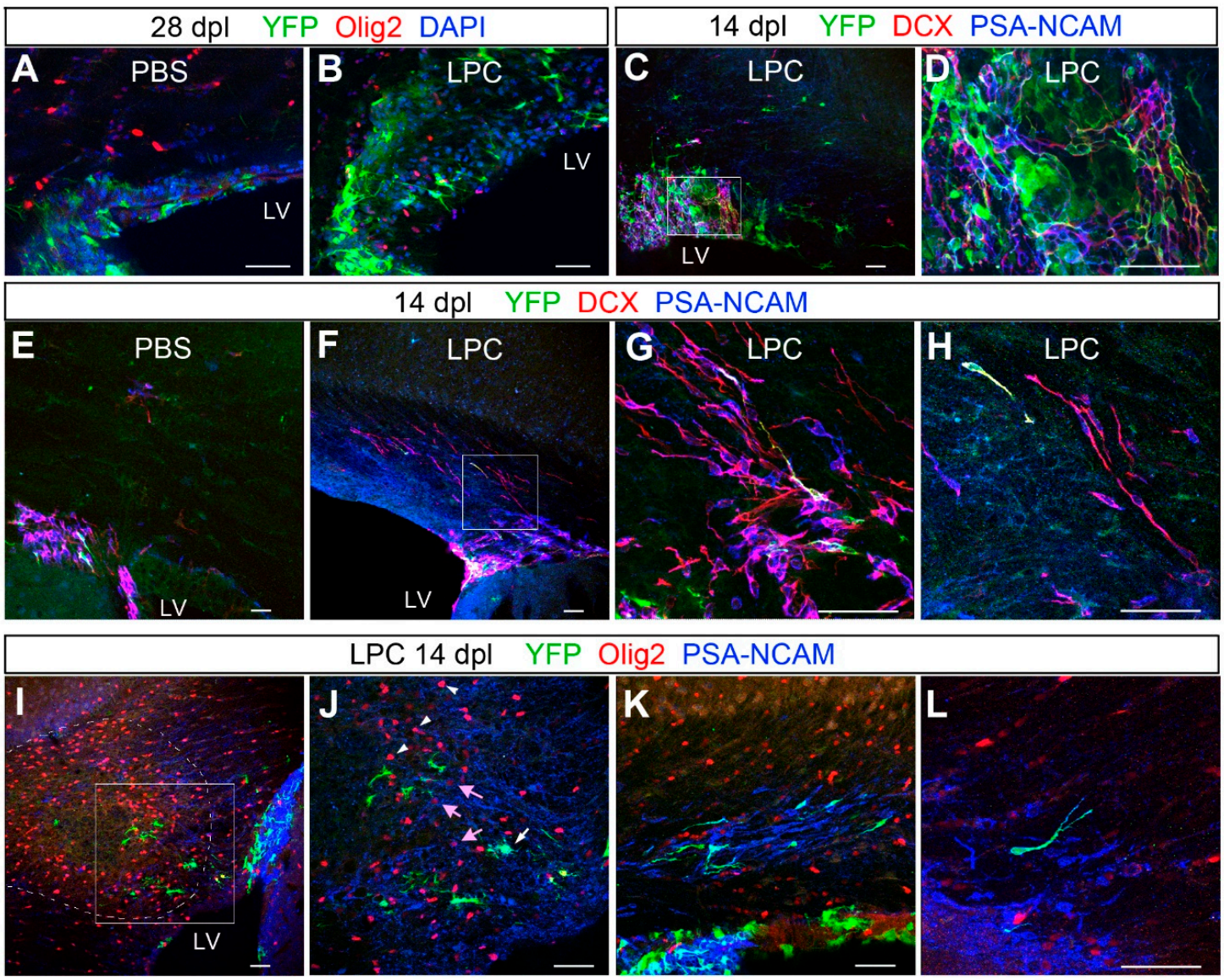

Figure 4. Changes in subventricular zone (SVZ) cells after LPC-induced demyelination. (A,B) Immunolabeling for YFP, Olig2, and DAPI showing increased thickness of the dorsal SVZ 28 days after LPC injection (B) compared with PBS-injected control (A). Very few Olig2+ cells are found in the control SVZ after PBS injection, whereas several Olig2+ cells are detected in the thickened SVZ after LPC injection. (C,D) Immunolabeling for doublecortin (Dcx) and PSA-NCAM showing that many of the YFP+ cells co-express Dcx and PSA-NCAM. (D) represents a higher magnification of the boxed area in C. (E-H) Immunolabeling for YFP, Dcx, and (PSA-NCAM) showing that most of the Dcx/PSA-NCAM+ cells are confined to the SVZ in control (E), whereas LPC-injected animals show a greater number of Dcx+ PSA-NCAM+ cells migrating dorsally into the SVZ $(\mathbf{F}-\mathbf{H})$. $(\mathbf{H})$ is a higher magnification image of the boxed area in (F). (I,J) Immunolabeling for YFP, Olig2, and PSA-NCAM near the lesion (dotted line) at $14 \mathrm{dpl}$. There is a dense cluster of PSA-NCAM+ cells in the lateral angle of the SVZ (right side in I). Higher magnification of the boxed area in (I), showing a YFP+ cell that is also PSA-NCAM+ ( $\mathbf{J}$, white arrow). There is a cluster of strongly Olig2+ cells inside the lesion (arrowheads in $(\mathrm{J})$ ), and weakly Olig2+ PSA-NCAM+ cells are detected at the lesion border (pink arrows in (J)). $(\mathbf{K}, \mathrm{L})$ PSA-NCAM+ cells, some of which are $\mathrm{YFP}+$, appear to be migrating in the corpus callosum in clusters $(\mathbf{K})$, and many of them have a tadpole-shaped unipolar morphology $(\mathbf{L})$. Scale bar: $50 \mu \mathrm{m}$.

To examine whether PSA-NCAM+ or Dcx+ cells contributed to the repair of the demyelinated lesion, we examined the phenotype and distribution of YFP+ PSA-NCAM+ cells in nestin-YFP mice after demyelination. At $14 \mathrm{dpl}$, many PSA-NCAM+ cells were found at the periphery of the lesion, while very few were seen in the core of the lesion where there was a high density of Olig2+ cells (Figure 4I,J). While the majority of the cells inside the lesion that were strongly positive for Olig2 did not express PSA-NCAM (Figure 4J, arrowheads), a few weakly Olig2+ cells around the lesion also expressed PSA-NCAM (Figure 4J, arrows). Most of the PSA-NCAM+ cells around the lesion were YFP-negative in nestin-YFP mice, suggesting that they had been generated from cells that were already neuroblasts or transit amplifying cells by the time of LPC injection. There were occasional 
clusters of PSA-NCAM+ cells oriented parallel to each other but not necessarily in the direction of the demyelinated lesion (Figure 4K,L). Some of the PSA-NCAM+ cells had the typical tadpole morphology, with their leading edge pointing away from the SVZ, suggestive of emigration from the SVZ into the corpus callosum. At $28 \mathrm{dpl}$, fewer than $2 \%$ of the PSA-NCAM+ cells expressed Olig2, and the majority of PSA-NCAM+ cells appeared to remain as migrating neuroblasts. These findings suggest that acute demyelination triggered emigration of PSA-NCAM+ Dcx+ neuroblasts from the SVZ. However, during the four weeks after demyelination, we did not detect a significant contribution of YFP+ PSA-NCAM+ cells into the lesion, even at $28 \mathrm{dpl}$ when we saw a significant number of YFP+ NG2+ cells in the corpus callosum. This makes it more likely that many of the NSC-derived OPCs that populated the corpus callosum after remyelination had directly differentiated into oligodendrocyte lineage cells from NSCs, rather than having passed through an intermediate stage of PSA-NCAM+ Dcx+ neuroblasts.

\section{Discussion}

We have shown that after chemically induced acute demyelination in the adult corpus callosum, local OPCs were rapidly recruited to the demyelinated lesion where they generated oligodendrocytes by $14 \mathrm{dpl}$. By contrast, nestin + SVZ cells expanded and migrated into the lesion but did so after a delay of two weeks and continued to generate OPCs in the corpus callosum through $28 \mathrm{dpl}$ when remyelination had already occurred. These observations suggest that local OPCs provide the major source of myelin repair after acute demyelination, while NSCs in the SVZ are an important source of repopulating the OPC population following their loss due to their differentiation into remyelinating oligodendrocytes.

\subsection{Oligodendroglial Fate of Local Parenchymal OPCs and NSCs in the SVZ}

It is well established that parenchymal OPCs that reside in the corpus callosum proliferate and generate remyelinating cells after an acute demyelinating injury created by local LPC injection [6], reviewed in $[1,33]$. There is also evidence that PSA-NCAM+ and/or Dcx+ neuroblasts in the SVZ migrate toward an acutely demyelinated lesion in the corpus callosum, although it has remained uncertain as to whether they generate remyelinating cells [16,18,34], see below).

The cuprizone-induced demyelinating model is another commonly used demyelination model with a more protracted time course. In mice fed on a cuprizone diet, demyelination occurs over five to six weeks, and remyelination ensues over the two to six weeks after the mice are returned to normal diet $[35,36]$. Moreover, if mice are continued on the cuprizone diet for 12 weeks, persistent demyelination occurs with poor remyelination [7]. Two recent studies used $\mathrm{Tg}\left(\mathrm{Nes}-\mathrm{creER}^{\mathrm{T} 2}\right)$ mice and $\mathrm{Tg}\left(\mathrm{Pdgfra}-\mathrm{creER}^{\mathrm{T} 2}\right)$ mice crossed to the ROSA-YFP reporter mice to compare the fate of NSCs and parenchymal OPCs, respectively, during the remyelination phase of cuprizone-induced demyelination $[19,20]$. In both studies, the progeny of NSCs, particularly those in rostral corpus callosum, robustly generated remyelinating oligodendrocytes to a greater extent than local OPCs. Furthermore, in regions above the SVZ, NSC-derived oligodendrocytes produced thicker myelin than local NG2 cell-derived oligodendrocytes [19].

On the contrary, another recent study used $\mathrm{Tg}\left(\mathrm{GFAP}-\mathrm{creER}^{\mathrm{T} 2}\right)$ crossed to the YFP reporter to examine the fate of GFAP+ NSCs in the SVZ following LPC-induced acute demyelination [21]. Despite a robust proliferative response in the SVZ in both the young and old mice, NSC-derived cells generated only a very small fraction of oligodendrocyte lineage cells, and the vast majority of oligodendrocyte lineage cells in the remyelinated lesion were generated from parenchymal cells [21], consistent with an earlier imaging study [37]. In our LPC-induced demyelination model, our observations were similar to the Kazanis study in that we found a greater contribution of local OPCs to oligodendrocyte regeneration but differed in that we saw a more significant influx of SVZ-derived OPCs by $28 \mathrm{dpl}$. 


\subsection{Temporal and Regional Determinants of Mobilizing Local OPCs and SVZ Cells}

The difference between our findings and those from the cuprizone studies may be partly attributed to the temporal difference in the evolution of myelin damage and repair in the cuprizone and LPC models. The more robust remyelination, mediated by the progeny of NSCs in the cuprizone model, could be because the SVZ has had time to expand during the prolonged two to six weeks of demyelination stage, and that two to seven days of demyelination in the LPC lesion was too short for these responses to occur in the SVZ. The increased density of NSC-derived OPCs in the lesioned corpus callosum that we observed at $28 \mathrm{dpl}$ is consistent with this, as is the observation that oligodendrocyte production from NSCs does not occur during the first four weeks [19]. Other studies that examined the response of more differentiated progeny of neural stem cells such as PSA-NCAM+ or Dcx+ neuroblasts in the SVZ have observed a more rapid response [16,18,32].

In the two cuprizone studies described above, the progeny of NSCs contribute significantly more to remyelination in the rostral corpus callosum, whereas local OPCs contribute more toward remyelination in the caudal corpus callosum. This could reflect the normal physiological dynamics of SVZ cells in rostral and caudal corpus callosum [38] and may reflect a greater abundance of oligodendrogliogenic progenitor cells in the caudal SVZ. In our experiments, LPC was injected into the rostral corpus callosum, and in most mice, demyelination spread laterally and caudally, so that the center of the demyelinated lesion typically occurred more than $500 \mu \mathrm{m}$ caudal from the injection site. Thus, our observation that local OPCs generated oligodendrocytes before NSC-derived cells did reflect the location of the demyelinated lesion. In addition to the rostro-caudal difference, there is medio-lateral heterogeneity among NSCs, those with oligodendroglial fate potential being more abundant along the dorsal SVZ than in the dorsolateral angle, where neurogenic NSCs reside [39].

\subsection{Plasticity of Neuronal and Oligodendrocyte Lineages in the SVZ}

The SVZ constitutes one of the two neurogenic niches in the mammalian CNS where neural stem cells reside and continue to generate new neurons and glia in the adult. Cells isolated from the adult mouse SVZ undergo self-renewal and can be induced to generate astrocytes, neurons, and a few oligodendrocytes $[14,39,40]$. Under normal physiological conditions, oligodendrocytes are a minor fate among the cells of the SVZ [41]. The adult mouse SVZ consists of nestin+ GFAP+ neural stem cells, PSA-NCAM+ Dcx+ neuroblasts that migrate through the RMS to the olfactory bulb, and rapidly amplify type C cells, which express Distal-less homeobox 2 (Dlx2) and give rise to neuroblasts [15,42]. In the SVZ, committed oligodendrocyte lineage cells are sparse, and importantly, OPCs are distinct from GFAP+ neural stem cells, rapidly proliferating type C cells, and Dcx+ neuroblasts [43].

After acute demyelination in the corpus callosum, PSA-NCAM+/Dcx+ progeny of NSCs, likely to be neuroblasts, extensively proliferate and migrate out of the SVZ toward the demyelinated lesion $[16,18]$. In response to an inflammatory lesion, the number of oligodendrocyte lineage cells increase in the SVZ at the expense of neurons in the olfactory bulb, resulting in impaired olfactory memory, suggesting plasticity of neuronal and glial fates of SVZ stem cells [17]. However, because oligodendrocyte lineage cells, particularly OPCs, actively transcribe low levels of neuronally expressed genes such as Dcx, Dlx2, and glutamic acid decarboxylase 67 (Gad1) [2], it has been a challenge to study the fate of neuroblasts or type $C$ cells $[18,43]$. Thus, it still remains unclear whether demyelination triggers reprogramming of normally neuronally committed NSCs or their progeny or amplifies oligodendrocyte-fated NSC cell clones.

\subsection{Mechanisms that Could Affect Oligodendrogliogenesis from the SVZ}

Local OPCs in the corpus callosum are capable of sensing a change in the degree of myelination or oligodendrocyte density and rapidly expand to repair the deficit [6,44]. Nestin+ NSCs, on the other hand, require two to three weeks to initiate the program to produce oligodendrocyte lineage cells, but given the necessary time, they can migrate and generate OPCs. How might a demyelinating lesion 
in the corpus callosum signal to the SVZ? Some proposed mechanisms include soluble factors such as netrin-1 [45] and epidermal growth factor [46] that promote emigration of cells out of the SVZ, and factors such as chordin [18], and Wnt7 [39] that promote oligodendroglial fate of NSCs. Besides these positive signals, negative regulators of oligodendrogliogenesis such as Gli1 [47], neurofibromin 1 [48], and Drosha [49] are beginning to be uncovered. How these mechanisms are coordinately regulated in response to demyelination remains to be elucidated.

\section{Conclusions}

In summary, we have shown that after acute demyelination in the corpus callosum, local OPCs rapidly expand and differentiate into remyelinating oligodendrocytes within the first two weeks. By contrast, NSCs in the SVZ begin their oligodendrogliogenic program with a temporal delay of two weeks, resulting in an increased population of SVZ-derived OPCs by four weeks after demyelination. The SVZ may be a limited source for repopulating OPCs, as it becomes depleted after sustained or repeated demyelination [20], which often occurs in chronic cases of multiple sclerosis (MS). Furthermore, the potential of SVZ-derived supply of OPCs in MS would be limited to lesions near the SVZ. Interestingly, NG2 cell density declines after remyelination of acutely demyelinated lesion in the spinal cord [3], while OPCs in the corpus callosum maintain their ability to self-renew even after prolonged cuprizone treatment for 12 weeks [20]. Further elucidation of the differences in the cellular properties of newly generated OPCs and those that have been residing in the white matter for an extended period of time could lead to new strategies to harness the ubiquitous population of local OPCs with enhanced ability for myelin repair.

Author Contributions: The study was conceived and developed by D.R.S. and A.N. D.R.S., S.S.P. and A.N. performed the experiments. A.L.R., S.S.P., C.C.B. and A.N. analyzed the data and performed the quantification. A.N. and D.R.S. wrote the manuscript.

Funding: The work was supported by grants from the National Institutes of Health (NIH) (R01NS049267, R01NS074870, and R01 NS073425 to AN), the National Multiple Sclerosis Society (RG4579A5/1 to AN), and Connecticut Stem Cell Program (06SCB03 to AN). The Leica SP8 confocal microscope was purchased using NIH Shared Instrumentation Grant S10OD016435 (PI, AN) and is maintained by Chris O'Connell, Director of Advanced Microscopy Facility.

Acknowledgments: We thank Youfen Sun for maintaining the mouse colony. We thank Amelia Eisch (University of Pennsylvania, Philadelphia, PA, USA) for the $\mathrm{Tg}\left(\mathrm{Nes}_{-} \mathrm{creER}^{\mathrm{T} 2}\right.$ ) mice and Tatsunori Seki (Tokyo Medical University) for the monoclonal 12E3 antibody to PSA-NCAM. We thank William Wood (University of Connecticut, Physiology and Neurobiology) for discussions and critical reading of the manuscript.

Conflicts of Interest: The authors declare no conflicts of interest.

\section{References}

1. Nishiyama, A.; Komitova, M.; Suzuki, R.; Zhu, X. Polydendrocytes (NG2 cells): Multifunctional cells with lineage plasticity. Nat. Rev. Neurosci. 2009, 10, 9-22. [CrossRef] [PubMed]

2. Nishiyama, A.; Boshans, L.; Goncalves, C.M.; Wegrzyn, J.; Patel, K.D. Lineage, fate, and fate potential of NG2-glia. Brain Res. 2016, 1638 (Pt B), 116-128. [CrossRef] [PubMed]

3. Keirstead, H.S.; Levine, J.M.; Blakemore, W.F. Response of the oligodendrocyte progenitor cell population (defined by NG2 labelling) to demyelination of the adult spinal cord. Glia 1998, 22, 161-170. [CrossRef]

4. Di Bello, C.I.; Dawson, M.R.; Levine, J.M.; Reynolds, R. Generation of oligodendroglial progenitors in acute inflammatory demyelinating lesions of the rat brain stem is associated with demyelination rather than inflammation. J. Neurocytol. 1999, 28, 365-381. [CrossRef] [PubMed]

5. Watanabe, M.; Toyama, Y.; Nishiyama, A. Differentiation of proliferated NG2-positive glial progenitor cells in a remyelinating lesion. J. Neurosci. Res. 2002, 69, 826-836. [CrossRef] [PubMed]

6. Gensert, J.M.; Goldman, J.E. Endogenous progenitors remyelinate demyelinated axons in the adult CNS. Neuron 1997, 19, 197-203. [CrossRef] 
7. Mason, J.L.; Toews, A.; Hostettler, J.D.; Morell, P.; Suzuki, K.; Goldman, J.E.; Matsushima, G.K. Oligodendrocytes and progenitors become progressively depleted within chronically demyelinated lesions. Am. J. Pathol. 2004, 164, 1673-1682. [CrossRef]

8. Dimou, L.; Simon, C.; Kirchhoff, F.; Takebayashi, H.; Gotz, M. Progeny of Olig2-expressing progenitors in the gray and white matter of the adult mouse cerebral cortex. J. Neurosci. 2008, 28, 10434-10442. [CrossRef] [PubMed]

9. Zhu, X.; Hill, R.A.; Dietrich, D.; Komitova, M.; Suzuki, R.; Nishiyama, A. Age-dependent fate and lineage restriction of single NG2 cells. Development 2011, 138, 745-753. [CrossRef] [PubMed]

10. Kang, S.H.; Fukaya, M.; Yang, J.K.; Rothstein, J.D.; Bergles, D.E. NG2+ CNS glial progenitors remain committed to the oligodendrocyte lineage in postnatal life and following neurodegeneration. Neuron 2010, 68, 668-681. [CrossRef] [PubMed]

11. Young, K.M.; Psachoulia, K.; Tripathi, R.B.; Dunn, S.J.; Cossell, L.; Attwell, D.; Tohyama, K.; Richardson, W.D. Oligodendrocyte dynamics in the healthy adult CNS: Evidence for myelin remodeling. Neuron 2013, 77, 873-885. [CrossRef] [PubMed]

12. Tripathi, R.B.; Rivers, L.E.; Young, K.M.; Jamen, F.; Richardson, W.D. NG2 glia generate new oligodendrocytes but few astrocytes in a murine experimental autoimmune encephalomyelitis model of demyelinating disease. J. Neurosci. 2010, 30, 16383-16390. [CrossRef] [PubMed]

13. Zawadzka, M.; Rivers, L.E.; Fancy, S.P.; Zhao, C.; Tripathi, R.; Jamen, F.; Young, K.; Goncharevich, A.; Pohl, H.; Rizzi, M.; et al. CNS-resident glial progenitor/stem cells produce Schwann cells as well as oligodendrocytes during repair of CNS demyelination. Cell Stem Cell 2010, 6, 578-590. [CrossRef] [PubMed]

14. Reynolds, B.A.; Weiss, S. Generation of neurons and astrocytes from isolated cells of the adult mammalian central nervous system. Science 1992, 255, 1707-1710. [CrossRef] [PubMed]

15. Doetsch, F.; Caille, I.; Lim, D.A.; Garcia-Verdugo, J.M.; Alvarez-Buylla, A. Subventricular zone astrocytes are neural stem cells in the adult mammalian brain. Cell 1999, 97, 703-716. [CrossRef]

16. Nait-Oumesmar, B.; Decker, L.; Lachapelle, F.; Avellana-Adalid, V.; Bachelin, C.; Van Evercooren, A.B. Progenitor cells of the adult mouse subventricular zone proliferate, migrate and differentiate into oligodendrocytes after demyelination. Eur. J. Neurosci. 1999, 11, 4357-4366. [CrossRef] [PubMed]

17. Tepavcevic, V.; Lazarini, F.; Alfaro-Cervello, C.; Kerninon, C.; Yoshikawa, K.; Garcia-Verdugo, J.M.; Lledo, P.M.; Nait-Oumesmar, B.; Baron-Van Evercooren, A. Inflammation-induced subventricular zone dysfunction leads to olfactory deficits in a targeted mouse model of multiple sclerosis. J. Clin. Investig. 2011, 121, 4722-4734. [CrossRef] [PubMed]

18. Jablonska, B.; Aguirre, A.; Raymond, M.; Szabo, G.; Kitabatake, Y.; Sailor, K.A.; Ming, G.L.; Song, H.; Gallo, V. Chordin-induced lineage plasticity of adult SVZ neuroblasts after demyelination. Nat. Neurosci. 2010, 13, 541-550. [CrossRef] [PubMed]

19. Xing, Y.L.; Roth, P.T.; Stratton, J.A.; Chuang, B.H.; Danne, J.; Ellis, S.L.; Ng, S.W.; Kilpatrick, T.J.; Merson, T.D. Adult neural precursor cells from the subventricular zone contribute significantly to oligodendrocyte regeneration and remyelination. J. Neurosci. 2014, 34, 14128-14146. [CrossRef] [PubMed]

20. Brousse, B.; Magalon, K.; Durbec, P.; Cayre, M. Region and dynamic specificities of adult neural stem cells and oligodendrocyte precursors in myelin regeneration in the mouse brain. Biol. Open 2015, 4, 980-992. [CrossRef] [PubMed]

21. Kazanis, I.; Evans, K.A.; Andreopoulou, E.; Dimitriou, C.; Koutsakis, C.; Karadottir, R.T.; Franklin, R.J.M. Subependymal zone-derived oligodendroblasts respond to focal demyelination but fail to generate myelin in young and aged mice. Stem Cell Reports 2017, 8, 685-700. [CrossRef] [PubMed]

22. Srinivas, S.; Watanabe, T.; Lin, C.S.; William, C.M.; Tanabe, Y.; Jessell, T.M.; Costantini, F. Cre reporter strains produced by targeted insertion of EYFP and ECFP into the ROSA26 locus. BMC Dev. Biol. 2001, 1, 4. [CrossRef]

23. Lagace, D.C.; Whitman, M.C.; Noonan, M.A.; Ables, J.L.; DeCarolis, N.A.; Arguello, A.A.; Donovan, M.H.; Fischer, S.J.; Farnbauch, L.A.; Beech, R.D.; et al. Dynamic contribution of nestin-expressing stem cells to adult neurogenesis. J. Neurosci. 2007, 27, 12623-12629. [CrossRef] [PubMed]

24. Schneider, C.A.; Rasband, W.S.; Eliceiri, K.W. NIH Image to ImageJ: 25 years of image analysis. Nat. Methods 2012, 9, 671-675. [CrossRef] [PubMed]

25. Trapp, B.D.; Peterson, J.; Ransohoff, R.M.; Rudick, R.; Mork, S.; Bo, L. Axonal transection in the lesions of multiple sclerosis. N. Engl. J. Med. 1998, 338, 278-285. [CrossRef] [PubMed] 
26. Miron, V.E.; Boyd, A.; Zhao, J.W.; Yuen, T.J.; Ruckh, J.M.; Shadrach, J.L.; van Wijngaarden, P.; Wagers, A.J.; Williams, A.; Franklin, R.J.; et al. M2 microglia and macrophages drive oligodendrocyte differentiation during CNS remyelination. Nat. Neurosci. 2013, 16, 1211-1218. [CrossRef] [PubMed]

27. Kucharova, K.; Stallcup, W.B. Distinct NG2 proteoglycan-dependent roles of resident microglia and bone marrow-derived macrophages during myelin damage and repair. PLoS ONE 2017, 12, e0187530. [CrossRef] [PubMed]

28. Seki, T.; Arai, Y. Highly polysialylated neural cell adhesion molecule (NCAM-H) is expressed by newly generated granule cells in the dentate gyrus of the adult rat. J. Neurosci. 1993, 13, 2351-2358. [CrossRef] [PubMed]

29. Doetsch, F.; Garcia-Verdugo, J.M.; Alvarez-Buylla, A. Cellular composition and three-dimensional organization of the subventricular germinal zone in the adult mammalian brain. J. Neurosci. 1997, 17, 5046-5061. [CrossRef] [PubMed]

30. Rutishauser, U.; Landmesser, L. Polysialic acid on the surface of axons regulates patterns of normal and activity-dependent innervation. Trends Neurosci. 1991, 14, 528-532. [CrossRef]

31. Rutishauser, U.; Landmesser, L. Polysialic acid in the vertebrate nervous system: A promoter of plasticity in cell-cell interactions. Trends Neurosci. 1996, 19, 422-427. [CrossRef]

32. Picard-Riera, N.; Decker, L.; Delarasse, C.; Goude, K.; Nait-Oumesmar, B.; Liblau, R.; Pham-Dinh, D.; Baron-Van Evercooren, A. Experimental autoimmune encephalomyelitis mobilizes neural progenitors from the subventricular zone to undergo oligodendrogenesis in adult mice. Proc. Natl. Acad. Sci. USA 2002, 99, 13211-13216. [CrossRef] [PubMed]

33. Franklin, R.J.; Ffrench-Constant, C. Remyelination in the CNS: From biology to therapy. Nat. Rev. Neurosci. 2008, 9, 839-855. [CrossRef] [PubMed]

34. Menn, B.; Garcia-Verdugo, J.M.; Yaschine, C.; Gonzalez-Perez, O.; Rowitch, D.; Alvarez-Buylla, A. Origin of oligodendrocytes in the subventricular zone of the adult brain. J. Neurosci. 2006, 26, 7907-7918. [CrossRef] [PubMed]

35. Blakemore, W.F. Observations on oligodendrocyte degeneration, the resolution of status spongiosus and remyelination in cuprizone intoxication in mice. J. Neurocytol. 1972, 1, 413-426. [CrossRef] [PubMed]

36. Ludwin, S.K. Central nervous system demyelination and remyelination in the mouse. Lab. Investig. 1978, 39, 597-612. [PubMed]

37. Guglielmetti, C.; Praet, J.; Rangarajan, J.R.; Vreys, R.; De Vocht, N.; Maes, F.; Verhoye, M.; Ponsaerts, P.; Van der Linden, A. Multimodal imaging of subventricular zone neural stem/progenitor cells in the cuprizone mouse model reveals increased neurogenic potential for the olfactory bulb pathway, but no contribution to remyelination of the corpus callosum. Neuroimage 2014, 86, 99-110. [CrossRef] [PubMed]

38. Luskin, M.B. Restricted proliferation and migration of postnatally generated neurons derived from the forebrain subventricular zone. Neuron 1993, 11, 173-189. [CrossRef]

39. Ortega, F.; Gascon, S.; Masserdotti, G.; Deshpande, A.; Simon, C.; Fischer, J.; Dimou, L.; Chichung Lie, D.; Schroeder, T.; Berninger, B. Oligodendrogliogenic and neurogenic adult subependymal zone neural stem cells constitute distinct lineages and exhibit differential responsiveness to Wnt signalling. Nat. Cell Biol. 2013, 15, 602-613. [CrossRef] [PubMed]

40. Gonzalez-Perez, O.; Alvarez-Buylla, A. Oligodendrogenesis in the subventricular zone and the role of epidermal growth factor. Brain Res. Rev. 2011, 67, 147-156. [CrossRef] [PubMed]

41. Maki, T.; Liang, A.C.; Miyamoto, N.; Lo, E.H.; Arai, K. Mechanisms of oligodendrocyte regeneration from ventricular-subventricular zone-derived progenitor cells in white matter diseases. Front Cell Neurosci. 2013, 7, 275. [CrossRef] [PubMed]

42. Komitova, M.; Zhu, X.; Serwanski, D.R.; Nishiyama, A. NG2 cells are distinct from neurogenic cells in the postnatal mouse subventricular zone. J. Comp. Neurol. 2009, 512, 702-716. [CrossRef] [PubMed]

43. Dayer, A.G.; Cleaver, K.M.; Abouantoun, T.; Cameron, H.A. New GABAergic interneurons in the adult neocortex and striatum are generated from different precursors. J. Cell Biol. 2005, 168, 415-427. [CrossRef] [PubMed]

44. Bu, J.; Banki, A.; Wu, Q.; Nishiyama, A. Increased NG2(+) glial cell proliferation and oligodendrocyte generation in the hypomyelinating mutant shiverer. Glia 2004, 48, 51-63. [CrossRef] [PubMed] 
45. Cayre, M.; Courtes, S.; Martineau, F.; Giordano, M.; Arnaud, K.; Zamaron, A.; Durbec, P. Netrin 1 contributes to vascular remodeling in the subventricular zone and promotes progenitor emigration after demyelination. Development 2013, 140, 3107-3117. [CrossRef] [PubMed]

46. Aguirre, A.; Dupree, J.L.; Mangin, J.M.; Gallo, V. A functional role for EGFR signaling in myelination and remyelination. Nat. Neurosci. 2007, 10, 990-1002. [CrossRef] [PubMed]

47. Samanta, J.; Grund, E.M.; Silva, H.M.; Lafaille, J.J.; Fishell, G.; Salzer, J.L. Inhibition of Gli1 mobilizes endogenous neural stem cells for remyelination. Nature 2015, 526, 448-452. [CrossRef] [PubMed]

48. Sun, G.J.; Zhou, Y.; Ito, S.; Bonaguidi, M.A.; Stein-O’Brien, G.; Kawasaki, N.K.; Modak, N.; Zhu, Y.; Ming, G.L.; Song, H. Latent tri-lineage potential of adult hippocampal neural stem cells revealed by Nf1 inactivation. Nat. Neurosci. 2015, 18, 1722-1724. [CrossRef] [PubMed]

49. Rolando, C.; Erni, A.; Grison, A.; Beattie, R.; Engler, A.; Gokhale, P.J.; Milo, M.; Wegleiter, T.; Jessberger, S.; Taylor, V. Multipotency of adult hippocampal NSCs in vivo is restricted by Drosha/NFIB. Cell Stem Cell 2016, 19, 653-662. [CrossRef] [PubMed]

(C) 2018 by the authors. Licensee MDPI, Basel, Switzerland. This article is an open access article distributed under the terms and conditions of the Creative Commons Attribution (CC BY) license (http://creativecommons.org/licenses/by/4.0/). 